\title{
A Hybrid Mining Model Based on Neural Network and Kernel Smoothing Technique
}

\author{
Defu Zhang, Qingshan Jiang, and Xin Li \\ Department of Computer Science, Xiamen University, 361005, China \\ dfzhang@xmu.edu.cn
}

\begin{abstract}
Neural networks as data mining tools are becoming increasingly popular in business. In this paper, a hybrid mining model based on neural network and kernel smoothing technique is developed. The kernel smoothing technique is used to preprocess data and help decision-making. Neural network is employed to predict the long trends of stock price. In addition, some trading rules involving trading decision-making are considered. The China Shanghai Composite Index is as case study. The return achieved by the hybrid mining model is four times as large as that achieved by the buy and hold strategy, so the proposed model is promising and certainly warrants further research.
\end{abstract}

\section{Introduction}

In this era of information blast, people meet huge amount of information everyday and are drowning in information. Information will provide huge profits for people if information can be utilized and processed, otherwise, it will become the burden of people. The goal of data mining is to bring the practice of information processing closer to providing the real answer and decision-making for the different investors and organizations. However, traditional data mining tools have been very difficult to meet the need of people. People have to find new computational models to get useful information from huge amount of data.

Neural networks (NNs) are a class of generalized nonlinear nonparametric models inspired by studies of the human brain. They are robust and have good learning and generalization capabilities [1], and are appropriate for clustering and prediction problems. For a more detailed description of NNs, the interested readers are referred to the papers in [2-4]. Due to NNs can mine valuable information from a mass of history information, so they have become one of the most efficient and useful mining tools [1, 5-8]. Especially, some researches [8-10] have shown that NNs performed better than conventional statistic approaches in financial forecasting. Despite NNs as data mining tool have many advantages, however, they still have some drawbacks, for example, overfitting and poor explanation capability and so on, which significantly affect the performance of NNs. In order to enhance the forecasting capability of NNs, many researchers have improved NNs by combining other techniques [11]. In this paper, the kernel smoothing technique, which is use to filter 'noise' and help decision-making, is combined with NNs to develop a mining model. The actual results show that this model is efficient, and some interesting results are obtained. 


\section{The Hybrid Mining Model}

Many researches [12] have shown that the closing price and the trading volume are the most important factors that affects stock market, so the two data are selected to predict the future trends of stock market. Due to the original financial data are very complex, and generally contain noise. In order to make model more effective, these data are usually preprocessed before training. In this paper, a kernel smoothing technique [12] is used to filter noise. In detail, for a time series $Y: y_{1}, \cdots, y_{n}$, a smoothed time series $\hat{Y}: \hat{y}_{1}, \cdots, \hat{y}_{n}$ is calculated as following:

$$
\hat{y}_{i}=\frac{\sum_{t=1}^{n} K_{h}(i-t) y_{t}}{\sum_{t=1}^{n} K_{h}(i-t)}, i=1,2, \cdots, n, K_{h}(x)=\frac{1}{h \sqrt{2 \pi}} e^{-\frac{x^{2}}{2 h^{2}}} .
$$

In addition, due to the activation function is a sigmoid function that squashes input data to $[0,1]$, so the two data are scaled in $[0.1,0.9]$ respectively.

The network architecture is shown in Fig.1.

\section{Input layer Hidden layer Output layer}

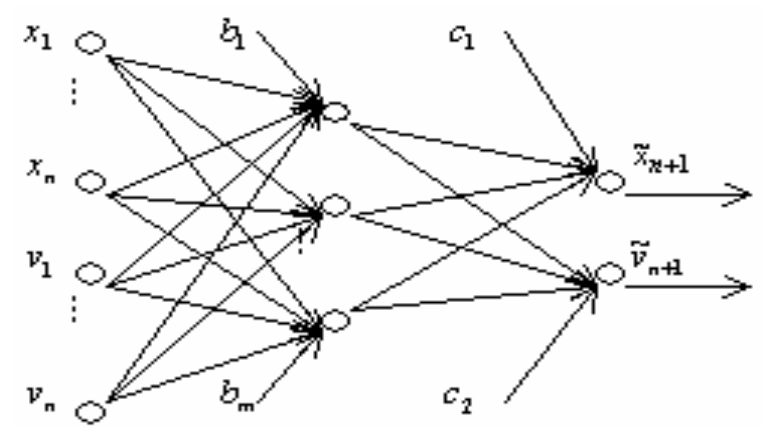

Fig. 1. A network architecture used by this paper

In order to make mode more effective, the following mean squared error is used.

$$
M S E=\frac{1}{2} \sum_{i=1}^{N} \alpha\left(\tilde{x}_{i+n}-x_{i+n}\right)^{2},
$$

where $\alpha=\left\{\begin{array}{cc}\beta & \text { if }\left(\tilde{x}_{i+n}-x_{i+n-1}\right)\left(x_{i+n}-x_{i+n-1}\right)>0 \\ 2-\beta & \text { else }\end{array}, \beta \in(0,1), N\right.$ is the total number of training pattern pairs, and $\tilde{x}_{i+n}$ is the computed output, $x_{i+n}$ is the target output. (1) denotes that the incorrect predicted directions are penalized more heavily than the correct predicted directions. 
In order to avoid the poor explanation capability of $\mathrm{NNs}$ and give valuable suggestions for our investment decision-making, some trading rules are considered. The trading rules 1 is as follows:

The trading signs $=\left\{\begin{array}{l}\text { Buy, } \quad \text { if } R_{B P}>R_{B T}, \\ \text { Sell, } \quad \text { if } R_{S P}<R_{S T}, \\ \text { Do nothing, otherwise. }\end{array}\right.$

where $R_{B P}=\frac{\max _{1 \leq i \leq 30}\left(\tilde{x}_{n+i}\right)-x_{n}}{x_{n}}, R_{S P}=\frac{\min _{1 \leq i \leq 30}\left(\tilde{x}_{n+i}\right)-x_{n}}{x_{n}}, R_{S P}$ and $R_{B P}$ are different constants and denote the return of predict selling and buying respectively.

All the seasoned investors know the stock trend is very important to make money, and the past trend will affect the future trend. Therefore, we obtain the trading rules 2 by combining the past trends and the trading rule 1 . Namely, the trading signs are sell if $R_{S P}<R_{S T}$ and the trend in the past two days is down. The trend can be obtained by the kernel smoothing techniques in the process of data preprocessing. We compare the return of the trading rule 1 with the return of the trading rule 2 (see Fig.2), the computational results have shown that the trading rule 2 is more effective. The mining model can tell us when to buy or sell the stock according to these rules.

\section{Computational Results and Conclusions}

The hybrid mining model was implemented in $\mathrm{C}++$ for Dos on a PC. The performance of this model was evaluated by trading the China Shanghai Composite Index from May 1996 to 15 Dec. 2003. The training patterns and test patterns are the data of 300 and 200 trading days respectively. Other parameters are designed as follows: $n=20, m=5, R_{B T}=0.06, R_{S T}=0.041, \beta=0.4$. The initial weights and thresholds are in $[-0.5,0.5]$. All the training patterns are selected randomly to train network about 3000 times, then all the testing patterns are tested. The process is repeated until the stop criteria are met.

The comparisons of returns between the mining model and the buy and hold strategy can be seen in Fig.2. All returns were calculated after taking the actual transaction costs for each transaction into consideration, namely the transaction costs for buying and selling is $1 \%$ respectively. For Fig.2, the return of the mining model with the trading rule 1 and 2 is 3.12 and 4.33 respectively, and the return of the buy and hold strategy is 1.31. The return of the trading rule 2 is about four times as large as that of the buy and hold strategy. So the mining model is superior to the buy and hold strategy. In addition, from Fig.2, it can know that the presented mining model performs better in bear market than in bull market, it denotes that the mining model has better capability of controlling risk. What is more, we find an interesting result, namely, the performance by training neural network every 150 days is better than that by training neural network every day. It directly improves the computational speed of the hybrid model.

The results of trading about seven years to Shanghai Composite Index have shown that the mining model was encouraging. This mining model can have actual application 


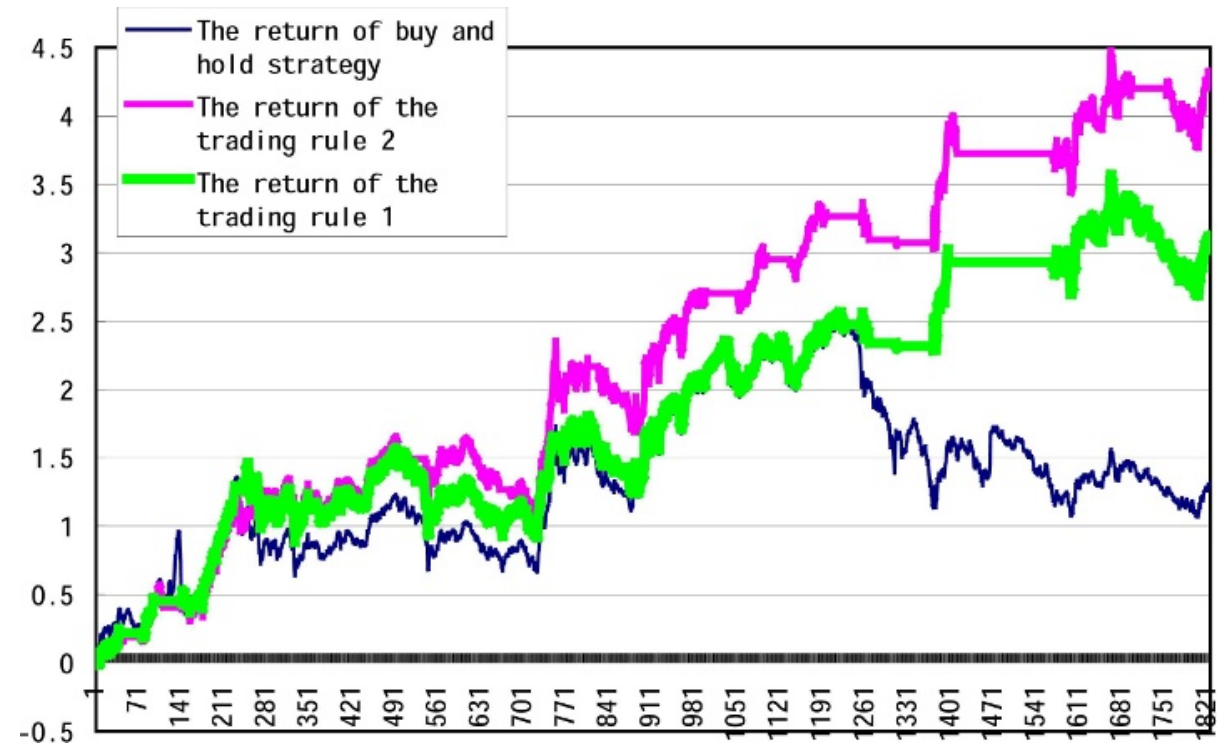

Fig. 2. Comparisons of returns between mining model and buy and hold strategy

and is very efficient for combinational investment. The future work is to strive for making the model more adaptive to the application and applying this model to other market and individual stocks.

\section{References}

1. Sushmita Mitra, Sankar K. Pal, and Pabitra Mitra: Data Mining in Soft Computing Framework: A Survey. IEEE Transactions on Neural Networks (2002) 13(1): 3-14

2. Hertz, J. Krogh, A., and Palmer R.G.: Introduction to the theory of neurocomputing. Addison-Wesley, Reading, MA (1991)

3. Widrow, B.; Rumelhart, D.E.; and Lehr, M.A.: Neural networks: applications in industry, business and science. Communications of the ACM (1994) 37(3): 93-105

4. Kate A.Smith, Jatinder N.D. Gupta: neural networks in business: techniques and applications for the operations researcher. Computers \& Operations Research (2000) 27:1023-1044

5. A. J. Chapman: Stock market trading systems through neural networks: Developing a model. International Journal of Applied Expert Systems (1994) 2(2): 88-100

6. Hoptroff R.: The principles and practice of time series forecasting and business modelling using neural nets. Neural Computing \& Applications (1993) 1: 59-66

7. Zhang G, Patuwo B, Hu M: Forecasting with artificial neural networks: the state of the art. International Journal of Forecasting (1998) 14: 35-62

8. Refenes,A.N., A.Zapranis, and G. Francis: Stock performance modeling using neural network: A comparative study with regression models. Neural Network (1994) 5: 961-970

9. Y.S.Abu-Mostafa, A.F. Atiya, M. Magdon-Ismail, and H. White: Neural networks in financial engineering. IEEE Transactions on Neural Networks (2001) 12(4): 653-656 
10. Saad E, Prokhorov E, Wunsch D.: Comparative study of stock trend prediction using time delay, Recurrent and probabilistic neural networks. IEEE Transactions on Neural Networks, (1998) 9:1456-70

11. Paul G. Harrald and Mark Kamstra: Evolving Artificial Neural Networks to Combine Financial Forecasts. IEEE Transactions on Evolutionary Computation 1997 1(1): 40-52

12. 12.Andrew W. Lo, Harry Mamaysky, Jiang Wang: Foundations of technical Analysis: Computational algorithms, statistical inference, and empirical implementation. http://www.nber.org/papers/w7613, National Bureau of Economic Research (2000) 